\title{
EL PROCESO DE APROBACIÓN DEL ÁREA PROTEGIDA MONTES DEL QUEGUAY CONFLICTOS, ACTORES, DESAFÍOS
}

\section{Verónica Filardo y Virginia Rossi}

\section{Resumen}

Las áreas protegidas son una herramienta de ordenamiento territorial privilegiada por los Estados para el control del uso del suelo y la sustentabilidad de los sistemas socioecológicos. En este artículo se presenta el estudio del proceso de integración de Montes del Queguay al Sistema Nacional de Áreas Protegidas, realizado con el objetivo de identificar los conflictos que se generan entre actores e intereses en torno a la apropiación de los servicios ecosistémicos en disputa. Mediante la realización de entrevistas semiestructuradas a informantes clave, se delimitó la periodización del proceso en tres fases (proyecto de solicitud de ingreso, delimitación del área y elaboración del plan de manejo] y se muestra cómo la configuración del conflicto y el posicionamiento de los actores varían en el tiempo.

Palabras clave: conflictos sociales, gobernanza, ordenamiento territorial, servicios ecosistémicos, temporalidad.

\begin{abstract}
The approval process for the protected area of Montes del Queguay. Conflicts, actors, challenges

Protected areas are a privileged territorial planning tool by the States for the control of land use and the sustainability of socio-ecological systems. This article shows the integration process of Montes del Queguay to the National System of Protected Areas study in order to identify the conflicts that are generated between actors and interests regarding the appropriation of the ecosystem services in dispute. Through the conduction of semi-structured interviews with key informants, the periodization of the process was delimited in three phases (admission application project, area delimitation and preparation of the management plan] and we show how the configuration of the conflict and the positioning of the actors vary in time.
\end{abstract}

Keywords: social conflicts, governance, land-use planning, ecosystem services, temporality.

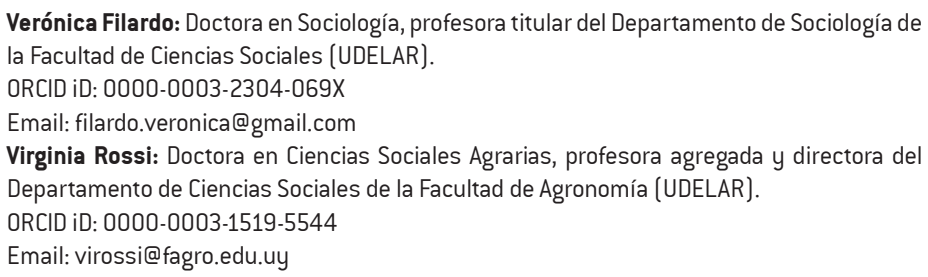




\section{Introducción}

Montes del Queguay, el territorio del estudio, está situado al noroeste del Uruguay, en el departamento de Paysandú. En 2014 fue declarado área protegida con recursos manejados e incluido en el Sistema Nacional de Áreas Protegidas (SNAP), organismo dependiente del entonces Ministerio de Vivienda y Ordenamiento Territorial y Medio Ambiente (MVOTMA).

Desde la década del ochenta del siglo XX, la reconfiguración de extensas áreas tradicionalmente ganaderas en el norte del país ha estado marcada por el avance de la silvicultura y la agricultura, predominantemente sojera, monocultivo que va de la mano de la expansión del modelo del agronegocio en la región (Fernandes, 2009; Rossi, 2010). En este sentido, organizaciones sociales de la zona de Guichón, junto con la Intendencia de Paysandú, presentaron en 2005 un proyecto de inclusión del área denominada Montes del Queguay para integrar al SNAP. En 2014, nueve años más tarde, se firmó el decreto que la constituye como área protegida bajo la figura "con recursos manejados". La inclusión de Montes del Queguay en el SNAP es excepcional, ya que parte de una solicitud de organizaciones sociales de la región, que fueron las que lideraron la iniciativa.

Las áreas protegidas no necesariamente implican evitar la tendencia general del capitalismo agrario de degradación de los recursos naturales (Santos, 2011). El proceso de consolidación de áreas protegidas no está exento de conflictos, dado que la conflictividad es un proceso constante alimentado por las contradicciones y desigualdades del capitalismo (Fernandes, 2008). El análisis del caso tiene particular interés desde la perspectiva de la gobernanza y del ordenamiento territorial. Estos instrumentos de protección y conservación de recursos naturales constituyen, paradigmáticamente, casos de ordenamiento y planeamiento de los territorios.

Montes del Queguay fue uno de los territorios del Uruguay seleccionados en el proyecto BEST-P (2015-2019), ${ }^{1}$ que trabajó desde la perspectiva de los servicios ecosistémicos y el ordenamiento territorial en cinco países de América Latina, con un equipo integrado por más de veinte investigadores formados en diversas disciplinas. El abordaje del trabajo para la región de Guichón y

1 Proyecto "Bridging ecosystem services and territorial planning (BEST-P): a southern South American initiative", del Instituto Interamericano para el Cambio Global (IAI-CRN 3095), coordinado por José Paruelo (Universidad de Buenos Aires). Más información disponible en $<$ http://www.iai.int/en/post/detail/crn-3095>. 
Montes del Queguay privilegió una mirada histórico-sociológica del proceso de constitución de un área protegida con recursos manejados en este territorio, los conflictos de intereses y las disputas por la apropiación de los servicios ecosistémicos que estuvieron presentes durante más de veinte años.

Si bien en este artículo se presentan resultados que provienen de una de las etapas del estudio realizado en Montes del Queguay y la región de Guichón, el trabajo supuso varias fases. En primer lugar (2017-2018), con el objetivo de elaborar el mapa de actores de la región de Guichón relativo a la integración del área protegida en el SNAP, se realizaron quince entrevistas semiestructuradas a diferentes agentes sociales, vinculados desde distintos ámbitos al proceso de constitución del Montes del Queguay (productores y colonos residentes del área, integrantes de organizaciones no gubernamentales [ONG] y de emprendimientos productivos de Guichón, instituciones públicas, técnicos del Instituto Plan Agropecuario [IPA], etcétera). Para la selección de los agentes se contó, además, con el antecedente del estudio realizado años antes sobre el capital social en la zona y la intervención de la UDELAR en proyectos de extensión (Rossi, 2007).

Las entrevistas produjeron un rico material y permitieron generar mapas de actores e identificar sus cambios y los de sus posiciones en diferentes momentos del proceso, que va desde el proyecto de solicitud de ingreso al SNAP hasta la conformación de la Comisión Asesora Específica (CAE) del área protegida, cuyo objetivo fundamental fue la elaboración del plan de manejo del área. El análisis de las entrevistas realizadas en la primera etapa de la investigación permitió identificar las categorías que se utilizan para clasificar las posiciones de los actores en cada una de las fases, así como las representaciones de los conflictos y las tensiones en las escalas local y regional. A su vez, permitió construir un universo de ideas a partir del cual, en una etapa posterior, se diseñaron las grillas de la metodología $\mathrm{Q}$, utilizadas para entrevistar a más de cuarenta informantes clave. Esto permitió construir una tipología de subjetividades sobre los cambios percibidos en el área protegida y su zona de influencia, cuyos resultados se discuten en otra publicación (Filardo y Gravina, 2020).

En este artículo se presentan reflexiones originadas en el análisis de las entrevistas semiestructuradas aplicadas a informantes clave, dado su nivel de involucramiento o de afectación con el área protegida. El material producido se organiza en torno al proceso vivido en el territorio, en tres fases identificadas por los entrevistados y que coinciden con las previstas en el protocolo de conformación de áreas protegidas del SNAP: 1) el proyecto para solicitar al SNAP el ingreso del área; 2) la delimitación del área a proteger y la definición de la figura de protección; y 3) la elaboración del plan de manejo. Debe destacarse que existen diferencias, en las tres fases, en las posiciones que ocupan los actores, vinculadas a los conflictos derivados del decreto de declaración como área protegida de recursos manejados Montes del Queguay (Decreto 343/014, del 25 de noviembre de 2014). 


\section{Antecedentes}

En 1992, Uruguay firmó el Convenio de Diversidad Biológica en la Cumbre de Río de 1992, ratificado por la Ley n. ${ }^{\circ} 16.408$ un año después, en la que se designó al MVOTMA como autoridad competente para su instrumentación y aplicación. Es a partir de ello que se creó el SNAP como principal institución para la preservación de la biodiversidad y el ordenamiento territorial rural. Con ello se pretendía:

"conciliar el cuidado del ambiente — en particular de la diversidad de paisajes, ecosistemas, especies y elementos culturales - con el desarrollo económico y social del país, apostando a generar oportunidades para las comunidades locales y la sociedad en su conjunto a través de la recreación, el turismo, la educación, la investigación y el desarrollo de actividades productivas compatibles con la conservación.” (DINAMA, s. f.).

En 2019 eran diecisiete las áreas ingresadas en el SNAP, con una superficie total de 334.125 hectáreas (incluyendo superficies terrestres y marinas). Si bien se estima que estas áreas comprenden a más del $70 \%$ de los paisajes existentes en Uruguay, su superficie total representa el 1,05\% del territorio total del país.

\section{Área protegida con recursos manejados Montes del Queguay}

\section{El territorio}

La denominación Montes del Queguay surge a partir del sitio conocido como Rincón de Pérez, donde confluyen los ríos Queguay Grande y Queguay Chico, en el departamento de Paysandú (Figura 1).

Figura 1. Ubicación del área Montes del Queguay

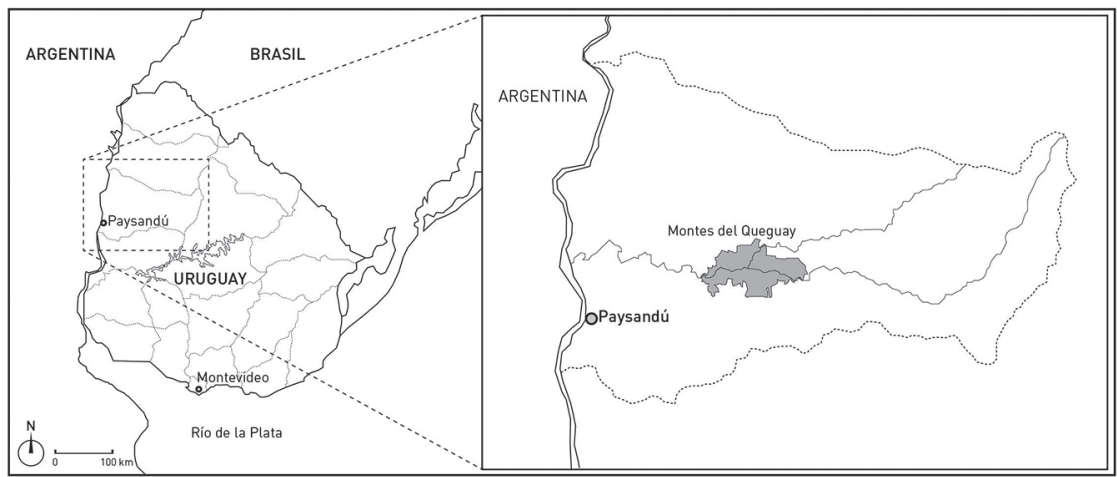

Fuente: Elaboración propia con base en MVTOMA, 2012. Diseño S. Cairus. 
El área protegida abarca 20.000 hectáreas,

“donde están representados diferentes ambientes: tierras bajas (bañados, pajonales, lagunas, cursos fluviales), una de las mayores masas de montes ribereños naturales del país, tierras altas (sierras basálticas y bosques de cerritos asociados), arbustales o chircales, bosques parque y pastizales no inundables, y se encuentran varias especies vegetales prioritarias para la conservación." (Decreto n. ${ }^{\circ}$ 343/014).

El territorio cuenta, además, con un relevante patrimonio cultural, histórico y arqueológico (tapera de Melchora, ${ }^{2}$ cercanía con Salsipuedes -el lugar de exterminio de los charrúas-, vestigios arqueológicos, etcétera).

En la región se encuentran grandes establecimientos dedicados fundamentalmente a la ganadería extensiva (vacuna y ovina), con algo de agricultura, en muchos casos en manos de extranjeros. A principios del siglo XXI, la expansión de la superficie dedicada a la soja se produjo a partir de un sistema de aparcería agrícola (medianería), en el cual el propietario de la zona, generalmente ganadero, arrienda pequeñas parcelas del establecimiento para un cultivo agrícola y va rotando año tras año, pues luego de la cosecha le dejan implantado un verdeo o una pradera permanente, mejoramiento que puede utilizar para la alimentación de su ganado (DINAMA, 2012). Otra actividad importante en la región es la apicultura (Achkar, Bessonart, Freitas y Melo, 2007).

La población de la región se concentra principalmente en Guichón, segundo centro poblado del departamento de Paysandú, y el resto se distribuye en pequeños centros poblados. La colonia Juan Gutiérrez, del Instituto Nacional de Colonización (INC), pertenece al territorio Montes del Queguay, aunque también hay otras colonias relativamente cercanas: Pintos Viana y José Batlle y Ordóñez. Las fracciones de la colonia Juan Gutiérrez tienen en promedio 300 hectáreas, fracciones que se consideran pequeñas debido a los índices de productividad del suelo, lo que lleva a que sus colonos sean considerados como "pequeños ganaderos" y registrados como "productores familiares" por las políticas públicas.

Las principales actividades en la zona son la ganadería, la agricultura, la lechería y, en menor escala, la forestación. Como fue mencionado, en la primera década del siglo XXI se registró un incremento de la producción de soja. Los predios de los colonos muestran síntomas de deterioro y han sido objeto de numerosos proyectos de recuperación de suelo en las últimas décadas, sobre todo por parte del IPA, como en forma recurrente se manifiesta en las entrevistas realizadas (DINAMA, 2009a). Por otra parte, el

2 Melchora Cuenca fue la mujer de José Artigas, héroe nacional. Ella y los hijos de ambos vivieron allí durante el exilio de Artigas en Paraguay. 
río Queguay hace posibles actividades náuticas y ecoturísticas que ponen en valor la conservación del paisaje.

\section{Sistema de actores}

Se presentan brevemente los resultados obtenidos en torno a los conflictos entre diferentes actores locales y regionales, en función de los intereses que proclaman y los servicios ecosistémicos que disputan. Se parte de quince entrevistas semiestructuradas realizadas a personas vinculadas al territorio en estudio e insertas en diferentes espacios de actuación. Algunos son jerarcas de organismos públicos o dirigentes de organizaciones sociales ambientalistas, otros están ligados a determinadas actividades económicas, como productores ganaderos, colonos del INC y técnicos de diferentes organismos públicos. Los entrevistados identificaron a los actores sociales que visualizan con participación en el proceso y a partir de este material se realizó un ordenamiento por parte del equipo de investigación y se elaboró un mapa de actores. Es de destacar que el protagonismo y la intensidad de la participación de algunos actores ha variado durante los años que van desde que se presentó la solicitud de inclusión de Montes del Queguay como área protegida hasta 2018.

El sistema de actores locales de la actualidad, tal y como fue construido a partir de las entrevistas, se presenta utilizando dos procedimientos diferentes. ${ }^{3}$ En primer lugar, se incluye un diagrama de Venn que coloca a los actores identificados en el momento actual en el territorio, las relaciones que mantienen entre sí (cooperación o conflicto) o la ausencia de vínculo. Se priorizó para ello la integración de la CAE, que formalmente oficia como espacio de gobernanza idóneo para la elaboración del plan de manejo.

En segundo lugar, se estableció un ordenamiento matricial de los actores (priorizados), en función del nivel de influencia y dependencia en la toma de decisiones en relación con el área protegida y, en consecuencia, las garantías sobre la provisión y límites para la apropiación de los servicios ecosistémicos del territorio. En la medida en que la figura de área protegida supone normativas para el uso del suelo, el control de los recursos naturales y la fiscalización de su cumplimiento, así como una gestión cogobernada y un plan de manejo establecido y diferenciado en zona núcleo y zona adyacente, se considera que la inclusión del área en el SNAP modifica las prácticas y produce cambios en el uso del suelo y el manejo, en extremo relevante. Estas transformaciones afectan a muchos actores locales y regionales. Sin embargo, el sentido de la afectación y el nivel de influencia en relación con las decisiones de este proceso no son iguales para todos. Existen quienes son más o menos afectados por dichos cambios y quienes tienen más o menos poder en la conducción de este proceso. En este sentido, para

3 Sobre la metodología de mapeo de actores, se recomienda ver Rossi, Filardo y Auer (2019) y Reed, Graves, Dandy, Posthumus, Hubacek, Morris, Prell, Quinn y Stringer (2009). 
ilustrar esta situación (Figura 3), no se consideran los actores públicos, sino exclusivamente aquellos actores de la sociedad civil o actores económicos.

Los objetivos que siguen ambas representaciones, si bien se complementan en la descripción de los intereses y conflictos en juego en el territorio, son diferentes. En el diagrama de Venn la pretensión es descriptiva y relacional, mientras que en la matriz de poder/dependencia los actores son distribuidos en función de su posición en una estructura que considera dos ejes o dimensiones (poder en las decisiones y dependencia de la conservación de los servicios ecosistémicos que viabiliza el área protegida).

Dado el carácter de la descripción de los actores en el territorio en estudio, definimos como actor central del área a la CAE. En el artículo 15 de la Ley n. ${ }^{\circ} 17.234$ se establece que:

"El Ministerio de Vivienda, Ordenamiento Territorial y Medio Ambiente constituirá, con relación a cada área natural protegida, una Comisión Asesora específica, en la que estarán representados el Poder Ejecutivo, los propietarios de predios privados incorporados al área, los pobladores radicados dentro del área, las autoridades locales y las organizaciones no gubernamentales ambientalistas con actividad vinculada al área." (Ley n. ${ }^{0} 17.234$ ).

La CAE de Montes del Queguay está integrada por la Dirección General de Promoción y Desarrollo y la Dirección de Desarrollo Rural de la Intendencia de Paysandú, delegados de MVOTMA, el SNAP, el Ministerio del Interior (MI), el Ministerio de Turismo (MINTUR) y el Ministerio de Ganadería, Agricultura y Pesca (MGAP), junto a pobladores, propietarios y ONG ambientalistas (Intendencia de Paysandú, 2016). Se presenta una breve caracterización de los actores en la Tabla 1.

Figura 2. Sistema de actores de Montes del Queguay, 2018

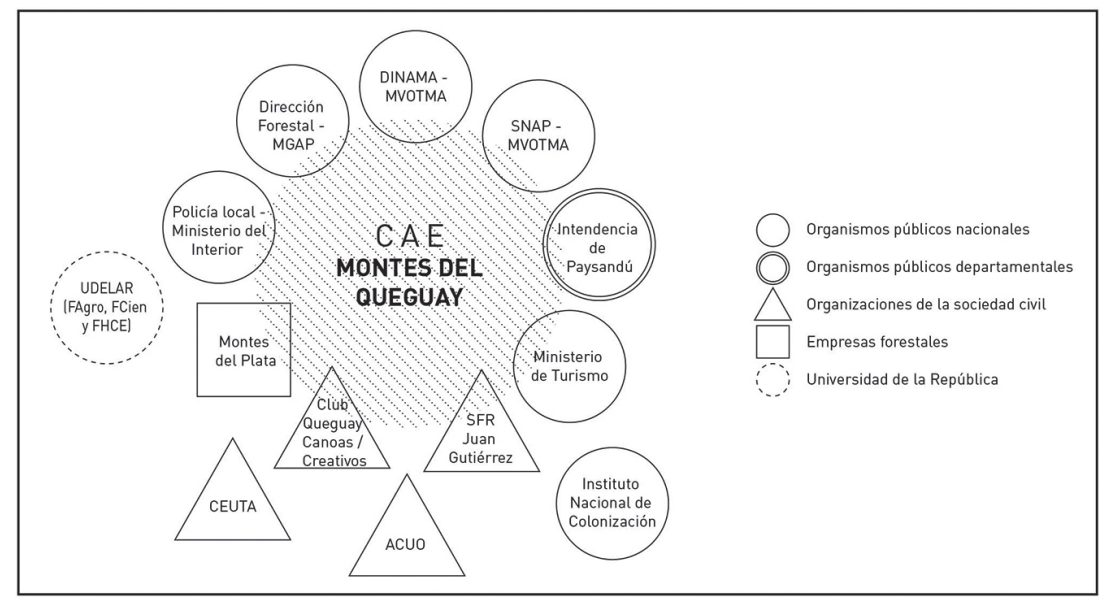

Fuente: Elaboración propia. Diseño: S. Cairus. 
Si bien se han priorizado algunos actores, existen otros que no fueron colocados en el diagrama, pero que también forman parte del sistema. Tal es el caso de la Junta Local de Guichón, que no integra la CAE. En la zona también funciona una mesa de desarrollo rural (MDR), un órgano de conformación interinstitucional y local, pero cuya actuación ha estado vinculada primordialmente con la colonia Juan Gutiérrez y no tiene mayor injerencia en las decisiones respecto al área protegida. Sin embargo, el funcionamiento de las MDR también contribuye a la consolidación de capital social en la zona, así como a dinámicas de trabajo colectivo y colaborativo. La Liga de Guichón agrupa a productores ganaderos grandes y tuvo peso relativo en épocas anteriores. Actualmente no participa de la CAE ni se configura como un actor relevante. Tampoco integran la CAE actores que fueron protagónicos en la fase de solicitud de ingreso del área protegida al SNAP, como la Asociación Conservacionista Uruguaya de Ornitología (ACUO) y el Centro Uruguayo de Tecnologías Apropiadas (CEUTA).

\section{Matriz de poder $y$ dependencia}

Para determinar las posiciones en torno a la dependencia de la conservación de los recursos ecosistémicos de Montes del Queguay y el nivel de influencia en la toma de decisiones para la elaboración del plan de manejo, se consideraron solo dos actores de la sociedad civil que participan actualmente en la CAE. Por ejemplo, el CEUTA es una organización de la sociedad civil de principios ambientalistas, con impronta académica y vocación científica. Aunque fue uno de los actores centrales en la formulación del proyecto de solicitud de ingreso de Montes del Queguay al SNAP, al inicio de ese largo proceso, no logró mantenerse en el territorio en el tiempo. No participa en la CAE y es un claro ejemplo de las variaciones en el mapa de actores en los diferentes momentos. Por este motivo, como se mencionó antes, no es incluido en el diagrama.

Se ha ubicado al Club Queguay Canoas como un actor social que tiene un interés específico en la protección del área, no solo por sus principios conservacionistas y de sustentabilidad ambiental, sino también porque la rentabilidad de sus actividades económicas depende de esto. En la medida que es una organización que realiza actividades vinculadas al ecoturismo y paseos histórico-patrimoniales, es imprescindible para su viabilidad asegurar que el río en el que se desarrolla el canotaje no esté contaminado, que pueda hacerse pesca recreativa sin pérdida de biodiversidad y que se mantengan el monte nativo, la belleza del paisaje, la fauna autóctona que permita avistamiento, etcétera. La conservación del ecosistema es condición sine qua non para estas actividades y emprendimientos. Por tanto, la dependencia respecto a la conservación de los servicios ecosistémicos que garantiza el área protegida para el Club Queguay Canoas, que se constituye hoy como el 
actor local más relevante, es muy alta. También es alto su poder de influencia, ya que participa en la CAE, ha tenido un rol protagónico (al inicio como parte de la Asociación Creativos) durante las tres fases del proceso y hoy es el interlocutor privilegiado de los organismos públicos que participan de la CAE y la contraparte de numerosos convenios para la realización de inversiones públicas en el territorio (como el Centro de Visitantes, con el MINTUR y con financiamiento del BID, entre otros).

Figura 3. Matriz de poder y dependencia de los actores en Montes del Queguay, 2018

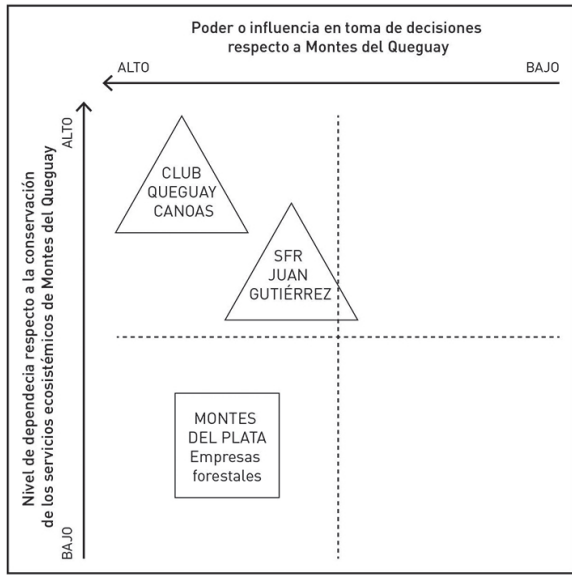

Fuente: Elaboración propia. Diseño: S. Cairus.

Montes del Plata, como gran empresa forestal de carácter internacional y que puede inscribirse en el modelo de agronegocio descrito por Fernandes (2009), tiene una baja dependencia en su estrategia productiva con respecto a la conservación de los recursos ecosistémicos de la zona. Por el contrario, la inclusión de Montes del Queguay como área protegida puede interpretarse como una iniciativa exitosa para frenar la expansión de la superficie forestada y controlar la presión de este rubro de explotación sobre el área. Sin embargo, la estrategia de posicionamiento de la empresa atiende a la cuestión ambiental y las medidas internacionalmente establecidas para minimizar el impacto negativo sobre el ecosistema que genera inevitablemente su implantación. Es posible que la participación de las empresas forestales en la CAE responda a esta estrategia, así como la medida de Montes del Plata de manejar parte de sus explotaciones como reservas naturales, con acciones que tomen en cuenta el cuidado ambiental y la investigación, lo que le asegura la producción forestal sin mayores controles en grandes superficies y garantiza sus ganancias. Su participación en la CAE convierte a esta empresa en un 
actor de gran influencia en la toma de decisiones, que no solo está dada por este motivo. Su tamaño, las regulaciones a las que está sujeta, pero también los privilegios de los que goza, le confieren capacidad de activar recursos que, en principio, son para su beneficio, aunque forman parte de los bienes públicos para el territorio (la obra de un tramo de la ruta 4, por ejemplo, tuvo significación para la circulación en la región). De igual manera, la mano de obra que absorbe la empresa en la zona hace que su poder en la toma de decisiones sea, de por sí, considerable.

La Sociedad de Fomento Rural Colonia Juan Gutiérrez también participa de la CAE. Pero esto si bien formalmente le otorga simetría con el resto de los actores, dista de asegurarla. Los productores de la colonia (particularmente aquellos cuyos predios se inscriben en el área) son los principales afectados por la conservación de los servicios ecosistémicos y las normativas que se establezcan en el plan de manejo, entre otras cosas porque su supervivencia depende de lo que "puedan hacer" (de lo que tengan permitido y de su capacidad de llevarlo a cabo). No obstante, debe destacarse que han sido parte del proceso desde su inicio, y su ingreso al área fue voluntario en la mayoría de los casos. Sin embargo, se atiende a una situación de vulnerabilidad mayor que la del resto de los actores, debida básicamente a sus condiciones materiales de existencia y su posicionamiento en la estructura productiva local. Son pequeños productores familiares con predios de 300 hectáreas promedio, de suelo basáltico, con obligaciones financieras permanentes (deben pagar la renta al INC, ya que son arrendatarios), pero son los que tienen mayor arraigo a la tierra en la zona. Los colonos actuales son la tercera generación de colonos que permanecen en el mismo predio del INC, lo que permite interpretar que la permanencia les da su "sentido de juego" (Bourdieu, 1988).

\section{Los conflictos en Montes del Queguay en el tiempo}

Las etapas que se describen a continuación definen no solo "momentos" diferentes en el proceso de la integración de Montes del Queguay en el SNAP (con objetivos y funciones específicas en dicho proceso, pautadas y reglamentadas: presentación del proyecto; delimitación del área y elaboración del plan de manejo), sino que, además, el protagonismo de los actores en cada una ellas también varía. El SNAP adquiere relevancia como institución fundamental a partir de la aprobación de Montes de Queguay como área protegida y no antes, por ejemplo. Los actores que se comprometen por el proyecto para la inclusión de Montes del Queguay en la reglamentación de área con recursos manejados también varían en el tiempo. En el material de campo se marca sistemáticamente un cambio en la significación que tiene la preservación de los recursos naturales para la población local, incluso para 
la localidad cercana de Guichón, en gran medida producto del alto número de proyectos e intervenciones de diferentes organismos en el territorio desde 2005. Para el plan de manejo, particularmente de la forma en que está siendo procesado y trabajado, se suman actores que no participaron en la formulación del proyecto, que van delineando nuevos conflictos y acciones estratégicas.

\section{Periodización}

El análisis de las entrevistas y el material documental consultado permite historizar el proceso de la integración de Montes del Queguay en el SNAP hasta 2019, identificando tres fases:

- Fase 1: La elaboración del proyecto de inclusión de Montes del Queguay como área protegida del SNAP por parte de organizaciones de la región, en alianza con la Intendencia de Paysandú.

- Fase 2: La delimitación del área a proteger y la definición de la figura de protección. Fue un proceso de negociación con los diferentes actores y sus diferentes intereses en la zona, en el que participan además diversos organismos públicos. Para esta fase se conformó una plataforma interinstitucional con representación de los actores locales de diferente tipo. El producto final de este proceso es el decreto de 2014 que define la figura de protección "con recursos manejados" (área protegida con recursos manejados Montes del Queguay) y delimita la extensión del área (establece los predios afectados del área núcleo y del área adyacente).

- Fase 3: La elaboración del plan de manejo. Una vez delimitada el área y aprobada por el SNAP, se abrió una nueva fase que definió la elaboración del plan de manejo del área, iniciada una vez aprobado el Decreto n. ${ }^{\circ} 343$ en 2014 y que continuaba en 2019.

La Tabla 1 describe los diferentes tipos de actores sociales de Montes del Queguay que fueron identificados en el sistema de actores en cada fase del proceso. 
Tabla 1. Breve caracterización de los actores y participación en las etapas del proceso de aprobación del área protegida, 2019

\begin{tabular}{|c|c|c|c|c|}
\hline Tipo de actor & Organización & Breve descripción & $\begin{array}{l}\text { Fases en } \\
\text { las que tuvo } \\
\text { presencia }\end{array}$ & $\begin{array}{l}\text { Integra la } \\
\text { CAE }\end{array}$ \\
\hline \multirow{7}{*}{$\begin{array}{c}\text { Organizaciones } \\
\text { de la sociedad } \\
\text { civil }\end{array}$} & $\begin{array}{l}\text { Sociedad de } \\
\text { Fomento Rural } \\
\text { Colonia Juan } \\
\text { Gutiérrez }\end{array}$ & $\begin{array}{l}\text { La organización ha participado en las } \\
\text { tres etapas identificadas. Los predios de } \\
\text { algunos colonos que la integran están } \\
\text { parcialmente afectados por la delimitación } \\
\text { del área protegida. En varios casos se trata } \\
\text { de la tercera generación de colonos en } \\
\text { estos predios. } \\
\text { Está conformada en su mayoría por } \\
\text { colonos de las colonias Juan Gutiérrez, } \\
\text { Pintos Viana y Batlle y Ordóñez, y también } \\
\text { por productores no colonos. }\end{array}$ & 1,2 y 3 & $\begin{array}{c}\text { Sí (dos } \\
\text { delegados) }\end{array}$ \\
\hline & $\begin{array}{l}\text { Club Queguay } \\
\text { Canoas }\end{array}$ & $\begin{array}{l}\text { Fue corresponsable del primer informe que } \\
\text { se realizó para proponer la zona como área } \\
\text { protegida. Está vinculado a actividades } \\
\text { de ecoturismo, canotaje, camping y } \\
\text { paseos turístico-ambientales e históricos. } \\
\text { Tiene sede en el Centro de Visitantes, } \\
\text { que es la puerta de entrada a Montes del } \\
\text { Queguay. Ha participado en las tres etapas } \\
\text { identificadas. }\end{array}$ & 1,2 y 3 & Sí \\
\hline & $\begin{array}{l}\text { Agrupación } \\
\text { Creativos }\end{array}$ & $\begin{array}{c}\text { Corresponsable del primer informe que } \\
\text { se realizó para proponer la zona como } \\
\text { área protegida. Integrada por actores de la } \\
\text { sociedad civil de Guichón. }\end{array}$ & 1 & No \\
\hline & $\begin{array}{l}\text { Organizaciones } \\
\text { con sede en } \\
\text { Guichón y en } \\
\text { Paysandú }\end{array}$ & $\begin{array}{l}\text { Hay un representante de Paysandú } \\
\text { Nuestro y Grupo Ecológico Naturista } \\
\text { Sanducero (GENSA) en la CAE. }\end{array}$ & 3 & Sí \\
\hline & $\begin{array}{c}\text { CEUTA } \\
\text { (con sede en } \\
\text { Montevideo) }\end{array}$ & $\begin{array}{l}\text { Tuvo un papel central en la promoción del } \\
\text { área protegida y realizó investigaciones } \\
\text { y estudios. Mantiene su impronta en } \\
\text { el proceso y es referenciada por los } \\
\text { entrevistados. Actualmente no integra la } \\
\text { CAE ni tiene un rol central en el territorio. }\end{array}$ & 1 & No \\
\hline & $\begin{array}{c}\text { ACUO } \\
\text { (con sede en } \\
\text { Montevideo) }\end{array}$ & $\begin{array}{l}\text { Fue una de las organizaciones sociales } \\
\text { que presentaron el proyecto de ingreso de } \\
\text { Montes del Queguay al SNAP. Actualmente } \\
\text { no tiene relevancia en el sistema de } \\
\text { actores y tampoco integra la CAE. }\end{array}$ & 1 & No \\
\hline & Vida Silvestre & $\begin{array}{l}\text { Colabora en la elaboración del plan de } \\
\text { manejo. }\end{array}$ & 3 & No \\
\hline
\end{tabular}




\begin{tabular}{|c|c|c|c|c|}
\hline Tipo de actor & Organización & Breve descripción & $\begin{array}{l}\text { Fases en } \\
\text { las que tuvo } \\
\text { presencia }\end{array}$ & $\begin{array}{l}\text { Integra la } \\
\text { CAE }\end{array}$ \\
\hline \multirow{5}{*}{$\begin{array}{l}\text { Organismos } \\
\text { públicos } \\
\text { de alcance } \\
\text { nacional } \\
\text { [ministerios, } \\
\text { entes públicos, } \\
\text { institutos no } \\
\text { estatales] }\end{array}$} & $\begin{array}{l}\text { DINAMA- } \\
\text { MVOTMA, } \\
\text { SNAP }\end{array}$ & $\begin{array}{l}\text { EI MVOTMA es coadministrador del área } \\
\text { junto con la Intendencia de Paysandú. La } \\
\text { DINAMA preside la CAE y actúa con medidas } \\
\text { directas de gestión en el entorno del área } \\
\text { protegida, articulando actividades y planes } \\
\text { de desarrollo en las áreas adyacentes. El } \\
\text { SNAP (creado en el MVOTMA a través de la } \\
\text { DINAMA) apunta a la gestión ambiental y a } \\
\text { la visualización de los beneficios del área } \\
\text { protegida por quienes son parte de estos } \\
\text { territorios. }\end{array}$ & 2 y 3 & $\begin{array}{c}\text { Sí [la } \\
\text { preside] }\end{array}$ \\
\hline & $\begin{array}{l}\text { Dirección } \\
\text { Forestal-MGAP }\end{array}$ & $\begin{array}{l}\text { Unidad ejecutora del MGAP que integra } \\
\text { la CAE y aporta en el asesoramiento y } \\
\text { control del uso sostenible de los recursos } \\
\text { naturales. }\end{array}$ & 2 y 3 & Sí \\
\hline & MINTUR & $\begin{array}{c}\text { Integra la CAE, apoyando específicamente } \\
\text { el desarrollo de infraestructura turística } \\
\text { orientada a la protección y conservación } \\
\text { de los valores naturales, históricos y } \\
\text { culturales del país. }\end{array}$ & 3 & Sí \\
\hline & $\begin{array}{l}\text { INC } \\
\text { Regional } \\
\text { Paysandú }\end{array}$ & $\begin{array}{l}\text { Vinculado al proceso mediante las } \\
\text { fracciones que integran el área protegida } \\
\text { con recursos manejados y los colonos } \\
\text { participantes en la Sociedad de Fomento } \\
\text { Rural Juan Gutiérrez. }\end{array}$ & 1,2 y 3 & $\begin{array}{l}\text { Sí (como } \\
\text { invitado) }\end{array}$ \\
\hline & $\begin{array}{l}\text { IPA } \\
\text { Regional Litoral } \\
\text { Norte }\end{array}$ & $\begin{array}{l}\text { Aunque no integra la CAE, sus técnicos } \\
\text { regionales han estado vinculados a todo } \\
\text { el proceso del área protegida, en particular } \\
\text { a través de proyectos de conservación } \\
\text { y mejoramiento del campo natural, } \\
\text { extensión, transferencia y capacitación en } \\
\text { producción ganadera. }\end{array}$ & 1,2 y 3 & No \\
\hline \multirow{4}{*}{$\begin{array}{l}\text { Organismos } \\
\text { públicos de } \\
\text { alcance local } \\
\text { (ejecutivo y } \\
\text { legislativo] }\end{array}$} & $\begin{array}{l}\text { Intendencia de } \\
\text { Paysandú }\end{array}$ & $\begin{array}{l}\text { Es coadministradora del área junto con el } \\
\text { MVOTMA. }\end{array}$ & $1,2,3$ & Sí \\
\hline & $\begin{array}{l}\text { Municipios } \\
\text { Piedras } \\
\text { Coloradas y } \\
\text { Guichón. Juntas } \\
\text { locales de El } \\
\text { Eucalipto y } \\
\text { Cerro Chato } \\
\end{array}$ & $\begin{array}{l}\text { Las juntas locales corresponden a los } \\
\text { municipios, el tercer nivel de gobierno } \\
\text { (local), según resulta de la Ley n. }{ }^{\circ} 19.272 \\
\text { (Ley de Descentralización y Participación } \\
\text { Ciudadana), aprobada en } 2014 \text {. Son citadas } \\
\text { a participar en todas las CAE, pero no se ha } \\
\text { logrado su concurrencia. }\end{array}$ & & No \\
\hline & $\begin{array}{l}\text { Junta } \\
\text { Departamental } \\
\text { de Paysandú }\end{array}$ & $\begin{array}{c}\text { Órgano legislativo que conjuntamente con } \\
\text { la Intendencia ejerce el gobierno dentro } \\
\text { de los límites del departamento. Participa } \\
\text { en la cogestión del Centro de Visitantes } \\
\text { del área. }\end{array}$ & & No \\
\hline & $\begin{array}{c}\text { Jefatura de } \\
\text { Paysandú (MI) }\end{array}$ & $\begin{array}{l}\text { Participa en la elaboración del plan de } \\
\text { manejo, principalmente en temas de } \\
\text { seguridad y fiscalización. }\end{array}$ & 3 & Sí \\
\hline
\end{tabular}




\begin{tabular}{|c|c|c|c|c|}
\hline Tipo de actor & Organización & Breve descripción & $\begin{array}{c}\text { Fases en } \\
\text { las que tuvo } \\
\text { presencia }\end{array}$ & $\begin{array}{c}\text { Integra la } \\
\text { CAE }\end{array}$ \\
\hline $\begin{array}{l}\text { Espacios de } \\
\text { gobernanza }\end{array}$ & $\begin{array}{c}\text { Mesa de } \\
\text { Desarrollo Rural } \\
\text { de Paysandú }\end{array}$ & $\begin{array}{l}\text { Ámbito de concertación territorial } \\
\text { (local-nacional). }\end{array}$ & & No \\
\hline Academia & $\begin{array}{l}\text { Facultades de } \\
\text { Agronomía, } \\
\text { Ciencias y } \\
\text { Humanidades } \\
\text { y Ciencias de } \\
\text { Educación } \\
\text { [UDELAR] }\end{array}$ & $\begin{array}{l}\text { Varias facultades participan de diversos } \\
\text { proyectos en el área protegida desde hace } \\
\text { al menos treinta años. Las Facultades } \\
\text { de Agronomía y Veterinaria han tenido } \\
\text { un intenso vínculo con los productores } \\
\text { de la zona en el formato de extensión } \\
\text { universitaria y mediante el desarrollo de } \\
\text { proyectos de investigación en el territorio. } \\
\text { La Facultad de Ciencias también ha llevado } \\
\text { adelante estudios que fueron insumos } \\
\text { importantes en la primera y la segunda } \\
\text { fase del proceso del decreto de } 2014 \text {. La } \\
\text { Facultad de Humanidades y Ciencias de } \\
\text { Educación, a través del Departamento de } \\
\text { Arqueología, ha generado importantes } \\
\text { antecedentes en torno a los vestigios } \\
\text { de pueblos aborígenes (charrúas) y los } \\
\text { hallazgos encontrados en el territorio. } \\
\text { El Polo de Ecología Fluvial trabaja } \\
\text { activamente en la formulación del plan de } \\
\text { manejo y en los trabajos de investigación } \\
\text { referidos a este. También participan en la } \\
\text { elaboración del plan otros integrantes del } \\
\text { Centro Universitario (CENUR) Litoral Norte. }\end{array}$ & 1,2 y 3 & No \\
\hline $\begin{array}{l}\text { Empresas } \\
\text { agropecuarias } \\
\text { y forestales }\end{array}$ & $\begin{array}{c}\text { Montes del } \\
\text { Plata y otras } \\
\text { empresas de la } \\
\text { zona }\end{array}$ & $\begin{array}{l}\text { Participan con dos delegados en la CAE. } \\
\text { De las empresas privadas de la zona, la } \\
\text { empresa forestal industrial más importante } \\
\text { es Montes del Plata, dedicada a la pulpa de } \\
\text { celulosa y fundada en Uruguay en } 2009 \\
\text { por Araucho (Chile) y Stora Enso (Suecia- } \\
\text { Finlandia). Si bien existieron diferentes } \\
\text { firmas dedicadas a la forestación con } \\
\text { eucaliptus en la zona, tuvieron al mismo } \\
\text { representante técnico durante las tres } \\
\text { etapas. }\end{array}$ & 1,2 y 3 & Sí \\
\hline
\end{tabular}

Fuente: Elaboración propia, revisado por Francisco Bergós [SNAP] en 2020.

\section{Fase 1: Proyecto de inclusión en el SNAP, conflictos iniciales}

Si bien el decreto de constitución del área protegida se firmó en 2014, las iniciativas de conservación de la zona datan de mediados del siglo XX. En 1949, algunos representantes de la comunidad de Guichón solicitaron la protección del llamado Rincón de Pérez. En 2006, diversas organizaciones sociales vinculadas a actividades turísticas y culturales (no agropecuarias), apoyadas por un proyecto de las Naciones Unidas (Proyecto Pequeñas 
Donaciones $)^{4}$ y acompañadas por la Intendencia de Paysandú, formalizaron la solicitud de inclusión de Montes del Queguay en el SNAP. A partir de allí, desde el SNAP se promovió la constitución de una unidad ejecutiva de carácter interinstitucional (llegó a convocar a más de treinta instituciones), que trabajó en la construcción de consensos locales para la protección del área. ${ }^{5}$ El 14 de diciembre de 2009, la DINAMA realizó la puesta de manifiesto público, donde se presentó el área protegida Montes del Queguay en el departamento de Paysandú en la categoría área protegida con recursos manejados (DINAMA, 2009b).

El análisis de las entrevistas realizadas revela que el principal conflicto identificado en el territorio en la primera etapa fue por el uso del suelo, fundamentalmente dedicado a algunos rubros de producción agropecuaria. De hecho, se enfrentó el avance de las grandes empresas trasnacionales de forestación y la introducción del cultivo de soja en agricultura. Ese fue el cambio más relevante en la reconfiguración productiva y social de la zona, que tradicionalmente ha sido "el corazón de la ganadería de Paysandú". El impacto sobre el ecosistema, tanto de la agricultura como de las forestales, era percibido como un riesgo, lo que en primera instancia reveló el enfrentamiento de dos posiciones: los "conservacionistas" y los "productivistas".

Así, en la primera etapa el conflicto se representa como polarizado. El argumento que se esgrime para legitimar esta clasificación puede formularse de la siguiente forma: mientras que para los conservacionistas los recursos naturales son valorizados en sí mismos y, por tanto, deben ser "conservados", para otros actores sociales la valorización de los recursos naturales está dada en función de su valor de uso, es decir, como factor de producción. En consecuencia, las medidas tendientes a la conservación son vistas por estos últimos como un "impedimento a la producción".

4 Proyecto PPD/FMAM/PNUD "Fortalecimiento de las comunidades locales para la gestión y uso sustentable de los recursos naturales asociados al río Quegua-í" (URY/06/02 noviembre de 2006-abril de 2008).

5 "En el marco de este proceso de trabajo que involucra un conjunto creciente de actores, en 2007 se constituyó el 'Plenario y Unidad Ejecutiva del área protegida', que se integra con referentes de la Intendencia Municipal de Paysandú; Sociedad de Fomento Rural de la Colonia Juan Gutiérrez; Agrupación Creativos; productores y propietarios del área; Club Queguay Canoas; CEUTA; Retos al Sur; Facultad de Agronomía; Universidad del Trabajo del Uruguay Guichón; Ministerio de Ganadería, Agricultura y Pesca; Ministerio de Vivienda, Ordenamiento Territorial y Medio Ambiente; Instituto Nacional de Colonización; Instituto Plan Agropecuario. Es en este ámbito que se lleva adelante el proceso de elaboración que constituye la base para la redacción de este borrador de proyecto" (DINAMA, 2009a, p. 2). 
Tabla 2. Representación del conflicto social en torno a Montes del Queguay en la primera fase

\begin{tabular}{ccc} 
& Conservacionistas & Productivistas \\
\hline Recursos naturales & Valor en sí, a conservar & Valor de uso para producir, para generar \\
& ganancia & geneficios \\
\hline Área protegida & Trabas, restricciones, impedimentos \\
\hline
\end{tabular}

El área protegida es vista como una herramienta del ordenamiento territorial que permite y asegura la conservación de los recursos naturales y como instrumento de control de los avances del agronegocio (representado por las empresas forestales y el incremento de la producción de soja, con intereses extranjeros), tendencia que se pretende revertir.

Si bien los técnicos entrevistados aluden al conflicto social respecto al área protegida de forma dicotómica: conservar versus producir ("conflicto estructural"), se ubican ellos mismos y al SNAP en una tercera posición, en el medio, "producir conservando":

"Creo que el más estructural y de fondo es el conflicto por el uso, entre una alternativa que es de usos naturales de conservación y usos productivos agropecuarios. Y ahí hay una gama de alternativas agropecuarias que son más compatibles con la conservación, pero, en definitiva, todas, incluso la ganadería extensiva, en un punto son incompatibles con la conservación [...]. Entonces, el conflicto estructural está ahí, entre la producción y la conservación." (Entrevista).

De hecho, los técnicos entrevistados sostienen que el principal trabajo de intervención con los productores del territorio afectado, como núcleo del área o adyacente, particularmente con los pequeños productores colonos del $\mathrm{INC}$, se orientó justamente a mitigar las posiciones polares y a abogar por la intermedia, favorecida y mediada por la propia figura en la que finalmente se inscribió el área protegida: "con recursos manejados": "Conservar implica producir con prácticas conservacionistas. Tenés que producir de una manera amigable con la conservación" (Entrevista).

Aunque el conflicto principal fue en esta etapa por el uso del suelo básicamente entre ganadería (con recursos manejados) y agricultura (soja) y forestación, los entrevistados identifican otros conflictos que se plantean con agentes sociales (que no se constituyen como actores, son "difusos"), como los cazadores furtivos y pescadores. Frente a ello, en la medida en que no es posible negociar ni "representar" esos intereses, la herramienta de la que se dispone es, en primer lugar, la legislación y la fiscalización (guardabosques), aunque también se menciona una conciencia social local que no favorece estas actividades ("no hay más baqueanos que acompañen a los cazadores"). 
Asimismo, se identifican conflictos entre diferentes actividades económicas, por ejemplo turismo o uso agropecuario, que están presentes desde el inicio (quienes presentaron el proyecto de inclusión del área en el SNAP no fueron los productores agropecuarios, sino organizaciones locales no vinculadas a la tenencia de la tierra). A su vez, el patrimonio cultural y arqueológico del territorio supone dificultades de gestión asociadas a la extranjerización de la tierra en la zona.

\section{Fase 2: Delimitación del área y figura de protección [2009-2014]}

La inclusión de un territorio en el SNAP requiere delimitar el área y la figura jurídica que regula el tipo de protección que le corresponde.

"Porque yo tengo un área protegida, Montes del Queguay que es donde se aplica el decreto estrictamente, un área adyacente donde no hay mucho para hacer, pero sí se trata de que se cumplan estrictamente las normas vigentes en otro tipo de cosas. Y después tengo otro territorio más grande, que es en el que trabajamos [...] sobre el paisaje circundante, tratando de que las áreas no queden como islas dentro de ese paisaje, sino que se incluyan en el paisaje y estén interactuando con ese paisaje." (Entrevista).

Es por eso que, en general, lo que se denomina "el área adyacente" no tiene las mismas exigencias en las condiciones de manejo de recursos que lo que se define como área protegida, y por esto también el proceso de la delimitación es negociado predio a predio. Luego de la intervención de diferentes organismos públicos y múltiples negociaciones, el área protegida quedó definida de la siguiente forma:

"El área protegida, en el caso específico de Montes del Queguay, está definida por ser una superficie de inundación que tiene que ver con todo el proceso hídrico de la región y que presenta un macizo boscoso que es el más grande del país. Esos son un poco los valores del área por los que se decidió incluirla dentro del SNAP. Dentro de esa área hay un complejo mosaico de distintos ecosistemas: lagunas, bañados, pastizales. Abarca unas mil hectáreas lo que es el área núcleo, el área donde realmente se aplica el decreto." (Entrevista).

El proceso de la delimitación del área fue una negociación de larga duración que intentó llegar a consensos, acordar con todos los implicados. Supuso resistencias, contemplaciones para con algunos agentes (propietarios de grandes establecimientos) y una definición que no profundizara los conflictos. Tuvo a favor que muchos quisieron entrar voluntariamente (algunos de los colonos de la colonia Juan Gutiérrez) y que otros aceptaron sin oponerse a la inclusión de parte de sus tierras (algunos grandes propietarios). 
Sin embargo, no se incluyó en el área protegida a los que tuvieron posiciones firmes sobre no querer ser afectados, dejándolos fuera de los límites del área.

"Los de la colonia quisieron entrar al área voluntariamente. El resto de la población, las estancias, no creo que quisieran entrar al área protegida. [...], todo el mundo pensaba que de entrar en el área protegida iban, a la corta o a la larga, a aparecer restricciones, 'esto no se puede hacer', entonces por eso no querían entrar. Sé que alguna estancia no presentó problemas ni nada, pero voluntariamente que quisieran entrar, no creo.” (Entrevista).

Se reitera en los relatos de los entrevistados sobre la fase de delimitación, la resistencia de los grandes propietarios a ser incluidos en el área. En el afán de consensuar y minimizar los conflictos, la DINAMA finalmente aprobó una delimitación de mínima, sin oposiciones de los afectados: "Y el proceso fue muy peleado, ahí fue que [...] muchos establecimientos se fueron enterando por escritos de El Telégrafo, pero fue muy peleado" (Entrevista).

$\mathrm{Si}$ bien se mantiene una cartografía del conflicto que enfrenta a los "conservacionistas" y a los "productivistas", aparecen en esta etapa significativas distinciones en ambos polos, en las representaciones de los entrevistados, que desdibujan la mirada simplista y dicotómica de la primera fase. Algunos entrevistados identifican diferencias entre los conservacionistas: por un lado, estaban aquellos "genuinamente interesados en la conservación"; por otro lado, quienes veían en la conservación beneficios económicos individuales y "disfrazados" (emprendimientos de ecoturismo).

"Ahí hay un núcleo de personas más genuinamente interesadas en la conservación. Después, había gente que estaba vinculada al turismo, y hasta el día de hoy está vinculada al turismo, que lo que quería era que toda esa zona... toda esa zona es propiedad privada, y también un recurso económico para el turismo, ellos antes no tenían acceso, hay un interés económico disfrazado. Todas esas tensiones se dieron en el territorio." (Entrevista).

Es importante señalar que las organizaciones sociales que presentaron el proyecto de inclusión de Montes del Queguay al SNAP en alianza con la Intendencia de Paysandú eran actores de la región, pero no eran propietarios ni tenían otra forma de tenencia sobre el suelo. Para algunos, el interés en la biodiversidad estaba dado, en alguna medida, porque se lucra con ella. Los emprendimientos de ecoturismo deben su ganancia a la conservación del ecosistema, y por ello en la regulación se juegan también intereses económicos privados. Muchas de las actividades de diferentes emprendimientos que funcionan en la zona están asociadas con el paisaje, el río, los montes, la fauna y la flora. 
"Gente vinculada a la cultura y al patrimonio de la zona presentó su proyecto y se hizo el trabajo de pedirle al SNAP que esté en el sistema. Pero no fueron productores del lugar, fueron actores de la región, actores que no eran los dueños de los lugares donde se estableció el área protegida. Se estableció un conflicto entre el uso económico de los productores agropecuarios y otros usos alternativos de esos lugares. Y el conflicto de intereses que hay detrás de eso." (Entrevista).

La representación del polo "productivista" como un agrupamiento que inicialmente aparecía compacto se desdibujó también a lo largo del proceso. Las diferencias entre los productores son notorias, se hicieron evidentes intereses y condiciones productivas muy desiguales, así como disposiciones hacia la conservación que no permiten sostener la clasificación simplista inicial. Es así que surgieron y se presentaron en las entrevistas nuevas formas de clasificación de los agentes sociales: el tamaño y las características de los predios diferencian sustantivamente a los colonos del INC de las grandes explotaciones ganaderas.

Los colonos, quienes en principio se veían como los más vulnerables respecto al impacto que las restricciones de producción y manejo podían generan en la viabilidad de sus explotaciones, mostraron mayor disposición a integrarse en el área, a partir de una actitud proclive a la protección sustentada en su vínculo con el ecosistema, su arraigo a la tierra y el paisaje, así como una alta carga emocional y compromiso moral. Propietarios de grandes explotaciones ganaderas con recursos patrimoniales significativos en el área también se comprometieron con el proyecto de protección de los servicios ecosistémicos, quebrando la homogeneidad pretendida de las etiquetas postuladas inicialmente. Los arrendatarios para la producción de soja vieron disminuidas sus posibilidades de desarrollo al cambiar las disposiciones de los posibles arrendadores, producto - no exclusivo- de un trabajo de concientización de varias instituciones públicas que fueron construyendo gradualmente alternativas productivas y de uso de la tierra y de manejo responsable de los recursos naturales.

Las representaciones de los conflictos y del posicionamiento de los actores en ellos fueron modificándose a medida que se avanzó en el proceso, atendiendo a una mayor complejidad. Es necesario, por otra parte, reconocer el efecto de la acción de actores externos al área (UDELAR, IPA), que cumplieron un papel central en la difusión de información de riesgos y en la generación y comunicación, vía la extensión universitaria, de alternativas productivas que promueven el cambio. 


\section{Fase 3: Elaboración del plan de manejo [2014-2019] ${ }^{6}$}

Montes del Queguay se encuentra en la etapa de elaboración del plan de manejo del área protegida, al haber ingresado al SNAP según el Decreto n. ${ }^{\circ}$ $343 / 014$, en el que se delimita y se inscribe en la figura concreta del área protegida con recursos manejados y se define, asimismo, la zona adyacente.

Para esta etapa, y con el objetivo explícito de elaborar el plan de manejo del área, se conformó la mencionada CAE.

"Por ahora no tenemos plan de manejo en el área, estamos trabajando en eso, pero lo único que tenemos es un decreto con una serie de restricciones al uso de esos recursos. Para la comunidad, para los dueños de la tierra, el ingreso del área fue un largo proceso de negociaciones con los tenedores de tierra, un proceso complejo con muchos obstáculos, pero se llegó a un cierto consenso para poder ingresarla en cuanto a los límites y en cuanto a la categoría para incluirla." (Entrevista).

La red de actores que se articula en torno al área protegida es densa y marcada por la presencia tanto de organizaciones sociales de diverso carácter, que representan diferentes intereses, como de un espectro amplio de instituciones públicas. A pesar de que, en algunos casos, la presencia de las organizaciones es intermitente y se identifican períodos en los que la participación es más activa que en otros, puede considerarse que la zona revela una actividad significativa en el proceso por parte de diversos grupos de interés y varios organismos nacionales, departamentales y locales. Por otra parte, la figura de la CAE, una vez que se disparó el proceso de la integración del área en el SNAP, obliga a una articulación entre la sociedad civil organizada, las empresas y los organismos del Estado, para que sean parte activa del proceso de toma de decisiones y definiciones normativas que constituirán el plan de manejo, $\mathrm{y}$, finalmente, para construir un sentido de totalidad e interés común. Todas estas son condiciones que favorecen el capital social del territorio, el reconocimiento mutuo que promueve la construcción colectiva de la identidad local y la gobernanza (Mayntz, 2001).

De las entrevistas se desprenden referencias que denotan un peso significativo dado a determinadas personas, vistas individualmente. Esto alude a empresarios o administradores de emprendimientos particulares que no siempre tienen el mismo signo en el posicionamiento respecto al área protegida: a algunos se les otorga carácter positivo y a otros no tanto. Asimismo, la impronta personal de determinados jerarcas de organismos públicos marca el proceso desde hace veinte años, con referencias cruzadas y reconocimientos mutuos. Particularmente, los técnicos han logrado

6 Se delimita la Fase 3 al momento de la elaboración del presente artículo. A octubre de 2020 se continuaba trabajando en esta fase. 
conformar una red personal y, mediada por ellos, institucional, que se activa como capital social para la dinámica del área protegida y del territorio en general. Este fenómeno es bastante común en Uruguay y se ha trabajado en torno a diferentes espacios de actuación pública (programas públicos de proximidad [Filardo, 2019], procesos de aprendizaje e innovación en el agro nacional [Filardo, 2001a; Rossi, Arbeletche y Courdin, 2011] y desarrollo local [Filardo, 2001b]).

Son las redes personales (sobre todo de técnicos y profesionales que ocupan cargos en la administración pública) las que en muchas oportunidades viabilizan las articulaciones interinstitucionales y las dinámicas territoriales (asimismo, podrían bloquearlas). En general, los lazos egocentrados se ponen a favor de los procesos colectivos en una suerte de red personal-institucional que se apoya fuertemente en liderazgos personales y alto compromiso con el tema convocante. Sin embargo, en las entrevistas se tensiona la capacidad de representación de quienes integran los espacios de participación establecidos. Se percibe que ocurre lo mismo con los cargos de representación de las organizaciones de la sociedad civil, que se entienden como legítimos en el ámbito de la negociación.

"Pasa con todos los lugares a los que uno va. Esas personas que te digo, con representación equilibrada en término de a qué sectores de interés representaban, porque teníamos dos ONG que representaban intereses ambientalistas, personas que representaban a los productores más chicos de la colonia, personas que representaban a instituciones que teníamos intereses en promover la conservación en estos lugares y personas que iban por los empresarios más grandes. Lo que yo veo que no hay es representación. Vos tenés personas que tienen esas sensibilidades que van supuestamente en representación de un colectivo más grande, pero con el que no necesariamente, en general, no hay posibilidad [...], se queda en la persona que va. Entonces creo que ha sido un proceso equilibrado desde ese punto de vista, pero muy divorciado de la masa que cada uno representaba." (Entrevista).

\section{Conclusiones}

La representación de los conflictos sociales en el área, así como la posición en que se ubican los diferentes actores locales y regionales, se han ido modificando en las diferentes etapas que supone normativamente la inclusión de Montes del Queguay como área protegida de recursos manejados del SNAP. $\mathrm{Al}$ inicio del proceso, en la fase de elaboración del proyecto para la inclusión del área, los entrevistados identifican dos posiciones antagónicas: quienes se colocan a favor de la protección, los "conservacionistas", y quienes rechazan el proyecto y sostienen intereses vinculados a la producción, "productivis- 
tas", en un conflicto que se enmarca como estructural por el uso del suelo. Sin embargo, una tercera posición, "intermedia", puede identificarse entre los técnicos entrevistados, orientados a defender el equilibrio entre ambos polos, fundamentada en "prácticas de producción amigables con el medioambiente" o con "producir conservando", que, a su vez, es compatible con la figura de protección en la cual finalmente se inscribió el área en el SNAP (de recursos manejados).

A medida que se avanzó en el proceso de delimitación del área y se empezaron a definir más claramente los intereses subyacentes a las posiciones, aparecieron distinciones dentro de lo que al inicio se dibujaba como posiciones polares para delinear el conflicto. Así, en las entrevistas se nombra a los "proteccionistas genuinos" y a aquellos que, bajo la consigna de la conservación, disfrazan intereses privados y beneficios económicos asociados a la protección de los recursos naturales, en gran medida agentes con emprendimientos ecoturísticos que no son propietarios de la tierra afectada ni residen dentro del área, sino en la localidad de Guichón. Por otra parte, la mayoría de los colonos de la colonia Juan Gutiérrez, cuyos predios total o parcialmente fueron incluidos en el área núcleo, ingresaron voluntariamente. Esto señala que aun quienes dependen de la producción agropecuaria valoran la conservación de los servicios ecosistémicos, incluso aunque las normativas del uso productivo del suelo y el manejo que se establecerá para el área protegida los desafíen para su sobrevivencia. Todo esto complejiza el mapeo del sistema de actores que en principio se representaba de forma simple.

La estrategia comunicacional de una de las empresas forestales (Montes del Plata) enfatiza su papel en la gestión de reservas naturales y la atención a medidas de cuidado medioambiental, al menos en parte de la superficie que explota. Montes del Plata forma parte de la CAE y la presencia de su representante es valorada positivamente por los otros integrantes, tanto del sector público como de las organizaciones de la sociedad civil.

La CAE constituye un espacio privilegiado de gobernanza instituido normativamente por el SNAP, en el que participan representantes de los organismos públicos involucrados, las empresas y la sociedad civil organizada. En la fase actual, corresponde a la CAE la elaboración y el ajuste del plan de manejo del área, un proceso en el que surgirán conflictos, debido a los diferentes intereses en juego en torno a la apropiación de los servicios ecosistémicos. No obstante la presencia de agentes que mantienen discrepancias, parece robusto el interés en la conservación, en gran medida porque buena parte de los directamente afectados (predios incluidos en el área) son colonos del INC con gran sentido de pertenencia, arraigo a la tierra e historia intergeneracional en esos predios. Manifiestan (de forma emocional y encarnada) su compromiso con el cuidado del medioambiente y creen que la expansión de la forestación y de la agricultura pone en riesgo el paisaje, la fauna y el ecosistema del Queguay. La gobernanza en la CAE de Montes 
del Queguay, entendida como la forma de gobierno en la que participan los diversos actores involucrados con capacidad de tomar decisiones con legitimidad y que, a pesar de antagonismos, logran cooperar, en el entendido de un sentido de totalidad que los trascienda, ha logrado consolidar el proyecto Montes del Queguay, generando capital social en el territorio en diferentes componentes (Grootaert y Van Bastelaer, 2001), en valores y normas compartidas y en regulaciones y disposiciones legales en las que participan a partir de la consolidación de espacios de decisión teóricamente horizontal de los intereses existentes en la región. La cooperación de actores externos, como la UDELAR, el IPA y otros, que han desarrollado proyectos de diferente orden considerando siempre a la población local como destinataria de sus intervenciones, ha contribuido en forma significativa al desarrollo de las condiciones necesarias para lo anterior.

En relación con la implementación de un instrumento de ordenamiento territorial, como lo son las áreas protegidas, el caso muestra la relevancia de estudiar los múltiples agentes-actores sociales que intervienen, así como aquellos que son afectados y las relaciones que mantienen entre sí, y, en particular, el papel del gobierno local como protagonista en el sostenimiento de la gobernanza en procesos de larga duración. Asimismo, pone de relieve el valor que adquiere comprender los "sentidos del juego" y las significaciones asociadas a los servicios ecosistémicos que configuran los conflictos. Por último, no puede estar ausente del análisis la dimensión temporal, en la medida que los conflictos sociales no son estáticos.

\section{Referencias bibliográficas}

Achkar, M.; M. Bessonart; G. Freitas y A. L. Mello (2007). Inclusión de los Montes del Queguay, departamento de Paysandú, en el Sistema Nacional de Áreas Protegidas. Montevideo: Facultad de Ciencias, UDELAR.

Bourdieu, P. (1988). Cosas dichas. Barcelona: Gedisa.

CEUTA (2008). Aportes para el plan de manejo del Área Protegida "Rincón de Pérez y el macizo de los montes del Queguay". Propuesta de los usuarios locales del Río Queguay. Paysandú: Grupo Creativos-CEUTA, PPD/GEF/ PNUD.

Decreto n. ${ }^{0} 343 / 014$ (2014). Aprobación de la selección y delimitación del área natural protegida "Montes del Queguay". Registro Nacional de Leyes y Decretos. Disponible en: <https://www.dinama.gub.uy/indicadores ambientales/wp-content/uploads/2015/09/Decreto-343_2014_Montes-delQueguay.pdf $>$ [acceso 20 de octubre de 2020] 
DINAMA(s.f.).Diversidadbiológica-Uruguay [en línea]. Disponible en: $<$ https:// www.cbd.int/iyb/doc/celebrations/iyb-uruguay-itinerantexposition-es.pdf> [acceso 20 de octubre de 2020].

DINAMA (2009a). Propuesta Proyecto de ingreso del área Montes del Queguay al Sistema Nacional de Áreas Protegidas. Versión 2. Diciembre. Montevideo: DINAMA-MVOTMA.

DINAMA (2009b). Puesta de Manifiesto Público Área Protegida "Montes del Queguay" en el Departamento de Paysandú bajo la categoría de "Área Protegidas con Recursos Manejados". Diario Oficial, 30 de diciembre. Disponible en: < https://impo.com.uy/diariooficial/2009/12/30/avisosdeldia. pdf $>$ [acceso 20 de octubre de 2020].

DINAMA (2012). Proyecto de selección y delimitación del área Montes del Queguay al Sistema Nacional de Áreas Protegidas. Montevideo: DINAMAMVOTMA. Disponible en: <https://www.gub.uy/ministerio-ambiente/ comunicacion/publicaciones/proyecto-ingreso-del-area-protegidarecursos-manejados-montes-del $>$ [acceso 20 de octubre de 2020].

Fernandes, B. M. (2008). Conflitualidade e desenvolvimento territorial. En A. M. Buainain (org.) (2008). Luta pela Terra, Reforma Agrária e Gestão de Conflitos no Brasil. San Pablo: Editora da UNICAMP. Disponible en: $<$ http:// web.ua.es/en/giecryal/documentos/documentos839/docs/bmfunesp-2.pdf> [acceso 20 de octubre de 2020].

Fernandes, B. M. (2009). Territorio, teoría y política. En F. Lozano Velásquez y J. G. Ferro Medina (eds.) (2009). Las configuraciones de los territorios rurales en el siglo XXI. Bogotá: Pontificia Universidad Javeriana, pp. 35-66.

Filardo, V. (2001a). Vínculos en los procesos de innovación e incorporación de biotecnologías en el agro de Uruguay [Tesis de Maestría en Sociología]. Montevideo: Facultad de Ciencias Sociales, UDELAR.

Filardo, V. (2001b). La dimensión económica y cultural en los procesos de desarrollo local [Tesis de Maestría en Desarrollo Local y Regional]. Montevideo: Universidad Católica del Uruguay.

Filardo, V. (2019). Las políticas de juventud de la izquierda en el Uruguay: ¿son “liberales”? En D. Beretta; F. Laredo; P. Núñez y P. Vommaro (comps.) (2019). Políticas de juventudes y participación política. Perspectivas, agendas y ámbito de militancia. Rosario: CLACSO, UNR Editora, 
pp. 247-262. Disponible en: <http://biblioteca.clacso.edu.ar/clacso/ gt/20191113060742/Politicas_de_juventud.pdf > [acceso 20 de octubre de 2020].

Filardo V. y V. Gravina (2020). Gobernanza y tipos de subjetividad de los actores sociales de Guichón en el área protegida Montes del Queguay. Agrociencia Uruguay, 24(1), pp. 1-15. Disponible en: <http://agrocienciauruguay.uy/ojs/ index.php/agrociencia/article/view/351> [acceso 20 de octubre de 2020].

Grootaert, C. y T. van Bastelaer (2001). Understanding and measuring social capital: a multidisciplinary tool for practitioners. Washington, DC: The World Bank Social Development Family Environmentally and Socially Sustainable Development Network. Social Capital Initiative. Disponible en: <https://openknowledge.worldbank.org/handle/10986/14098> [acceso 20 de octubre de 2020].

IAI (s. f.). BEST-P: Bridging ecosystem services and territorial planning, a southern South American initiative (CRN 3095) [en línea]. Disponible en: <http://www.iai.int/en/post/detail/crn-3095> [acceso 20 de octubre de 2020].

Intendencia de Paysandú (2016). Intendencia participa en la CAE del Área Protegida Montes del Queguay [en línea]. Disponible en: <https:// paysandu.gub.uy/departamento-de-descentralizacion/lorenzo-geyres/2851intendencia-participa-en-la-comision-asesora-del-area-protegida-montesdel-queguay $>$ [acceso 20 de octubre de 2020].

Ley n. ${ }^{\circ} 16.408$ (2000). Declaración de Interés General. Sistema Nacional de Áreas Naturales Protegidas. Diario Oficial, 9 de marzo. Disponible en: <https://www.impo.com.uy/bases/leyes/17234-2000> [acceso 20 de octubre de 2020].

Mayntz, R. (2001). El Estado y la sociedad civil en la gobernanza moderna. Revista del CLAD. Reforma y Democracia, 21 [en línea]. Disponible en: $<$ http://old.clad.org/portal/publicaciones-del-clad/revista-clad-reformademocracia/articulos/021-octubre-2001/0041004> [acceso 20 de octubre de 2020].

Reed, M. S.; A. Graves; N. Dandy; H. Posthumus; K. Hubacek; J. Morris; C. Prell; C. H. Quinn y L. C. Stringer (2009). Who is in and why? A typology of stakeholder analysis methods for natural resource management. Journal of Environmental Management, 90(5): 1933-1949. 
Rossi, V. (2007). Los proyectos de extensión universitaria y la construcción de capital social en la zona Guichón [Tesis Maestría en Ciencias Agrarias]. Montevideo: Facultad de Agronomía, UDELAR. Disponible en: < https:// www.colibri.udelar.edu.uy/jspui/bitstream/20.500.12008/1803/1/0011 ros. pdf $>$ [acceso 20 de octubre de 2020].

Rossi, V. (2010). Territorios en conflicto. Reestructuración productiva y producción familiar en el campo uruguayo. Revista Pampa, 6(6), pp. 89111.

Rossi, V.; P. Arbeletche y V. Courdin (2011). La Mesa del Queso Artesanal del Litoral como Innovación Organizacional para la Articulación Local de Políticas Públicas en Uruguay. Revista Estudios Cooperativos, 16(1), pp. 118-130. Disponible en: <https://www.extension.udelar.edu.uy/wp-content/ uploads/2015/10/05_Revista_Estudios_Cooperativos_vol_16_-_No_1__2011.pdf $>$ [acceso 20 de octubre de 2020].

Rossi, V.; V. Filardo y A. Auer (2019). Dispositivo metodológico para analizar el sistema de actores de un territorio. En J. Paruelo y P. Laterra (eds.) (2019). El lugar de la naturaleza en la toma de decisiones. Servicios Ecosistémicos y Ordenamiento Territorial Rural. Buenos Aires: Ediciones CICCUS, pp. 246-252.

Santos, C. (2011). Sustentabilidad, desigualdades sociales y justicia ambiental en el entorno de un área protegida. Revista Estudios Cooperativos, 16(1): pp. 104-117. Disponible en: <https://www.extension.udelar.edu.uy/wp-content/ uploads/2015/10/05_Revista_Estudios_Cooperativos_vol_16_-_No_1__2011.pdf $>$ [acceso 20 de octubre de 2020].

\section{Contribución de autoría}

Este trabajo fue realizado en partes iguales por Verónica Filardo y Virginia Rossi. 